\title{
Cancer Metabolism: New Validated Targets for Drug Discovery
}

\author{
Federica Sotgia ${ }^{1}$, Ubaldo E. Martinez-Outschoorn ${ }^{2}$, and Michael P. Lisanti ${ }^{1}$ \\ ${ }^{1}$ Manchester Breast Centre \& Breakthrough Breast Cancer Research Unit, Faculty Institute of Cancer Sciences, University \\ of Manchester, UK \\ ${ }^{2}$ Kimmel Cancer Center, Thomas Jefferson University, Philadelphia, PA \\ Correspondence to: Federica Sotgia, email: fsotgia@gmail.com
}

Ubaldo E. Martinez-Outschoorn, email: Ubaldo.Martinez-Outschoorn@jeffersonhospital.org

Michael P. Lisanti, email: michaelp.lisanti@gmail.com

Keywords: cancer metabolism; therapeutic targets; drug discovery; oncogenes; tumor suppressors; oxidative stress; glycolysis; cancer associated fibroblast; tumor microenvironment; metabolic symbiosis; anti-angiogenic therapy

Received: July 15, $2013 \quad$ Accepted: July 21, $2013 \quad$ Published: July 22, 2013

This is an open-access article distributed under the terms of the Creative Commons Attribution License, which permits unrestricted use, distribution, and reproduction in any medium, provided the original author and source are credited.

\section{ABSTRACT:}

Recent studies in cancer metabolism directly implicate catabolic fibroblasts as a new rich source of $i$ ) energy and ii) biomass, for the growth and survival of anabolic cancer cells. Conversely, anabolic cancer cells upregulate oxidative mitochondrial metabolism, to take advantage of the abundant fibroblast fuel supply. This simple model of "metabolic-symbiosis" has now been independently validated in several different types of human cancers, including breast, ovarian, and prostate tumors. Biomarkers of metabolic-symbiosis are excellent predictors of tumor recurrence, metastasis, and drug resistance, as well as poor patient survival. New pre-clinical models of metabolic-symbiosis have been generated and they genetically validate that catabolic fibroblasts promote tumor growth and metastasis. Over 30 different stable lines of catabolic fibroblasts and $>\mathbf{1 0}$ different lines of anabolic cancer cells have been created and are well-characterized. For example, catabolic fibroblasts harboring ATG16L1 increase tumor cell metastasis by $>11.5$-fold, despite the fact that genetically identical cancer cells were used. Taken together, these studies provide $>40$ novel validated targets, for new drug discovery and anti-cancer therapy. Since anabolic cancer cells amplify their capacity for oxidative mitochondrial metabolism, we should consider therapeutically targeting mitochondrial biogenesis and OXPHOS in epithelial cancer cells. As metabolic-symbiosis promotes drug-resistance and may represent the escape mechanism during anti-angiogenic therapy, new drugs targeting metabolic-symbiosis may also be effective in cancer patients with recurrent and advanced metastatic disease.

Metabolic-symbiosis represents a paradigm shift in cell biology and cancer metabolism [1-20]. In this simple metabolic model, catabolic fibroblasts fuel the growth of adjacent anabolic cancer cells, via energy transfer (Figure 1) $[2-4,7,12,13,15,17,19-53]$. Catabolic stromal cells produce high-energy mitochondrial "biofuels", such as L-lactate, ketone bodies, glutamine, other amino acids, and free-fatty acids, for cancer cells to use as substrates for OXPHOS and as biomass. [38, 40].

Catabolic fibroblasts also show a pro-inflammatory phenotype, due to oxidative stress and NFkB activation, which leads to cytokine production. This, in turn, attracts and serves to activate inflammatory cells (macrophages and neutrophils), which produce more ROS and hydrogen peroxide species. These findings link inflammation directly with energy transfer to anabolic cancer cells [2, $41,42,54,55]$, explaining how inflammation energetically promotes tumor initiation and cancer progression.

To stringently test the validity of these energy transfer mechanism(s), stable cell lines of constitutively catabolic fibroblasts were generated by genetically increasing glycolysis, ketogenesis, autophagy, mitophagy, oxidative stress, and/or senescence. This was accomplished by the recombinant over-expression or knock-down of key 
metabolic target genes in hTERT-immortalized fibroblasts. Similar results were obtained by the genetic manipulation of either growth factors or extracellular matrix proteins, indicating that these "signaling networks" also converge on catabolic metabolism in stromal fibroblasts.

These results are summarized in Table 1, which lists nearly 30 catabolic fibroblast cell lines that have been generated, to date [1-20]. Remarkably, these catabolic fibroblasts [56] effectively promoted tumor growth and/ or metastasis, in pre-clinical animal models (xenografts in nude mice) [1-20]. Similar results have also been obtained by using a syngeneic orthotopic animal model, employing the mammary fat pads of Cav-1 (-/-) null mice, as the catabolic host microenvironment for tumor growth [57].

Conversely, over-expression of metabolic genes that drive increased mitochondrial biogenesis or OXPHOS in epithelial cancer cells, also effectively promoted tumor growth, and induced autophagy-resistance (Table 1) $[4,8$, 18-20].

As metabolic-symbiosis may represent the underlying basis of drug-resistance [31, 32], and/or the escape mechanism $[35,43,44,47,48]$ during antiangiogenic therapy [53], new drugs that target metabolicsymbiosis may prove to be effective in patients with recurrent cancers and even for the treatment of advanced metastatic disease [25-27, 35, 43].

The existence of metabolic-symbiosis (a.k.a., twocompartment tumor metabolism) has also been directly validated in human breast cancer tissue sections, by employing mitochondrial activity staining in situ. Using this approach, it is clear that oxidative, mitochondrialrich cancer cell nests, are physically surrounded by

\section{Two-Compartment Tumor Metabolism}

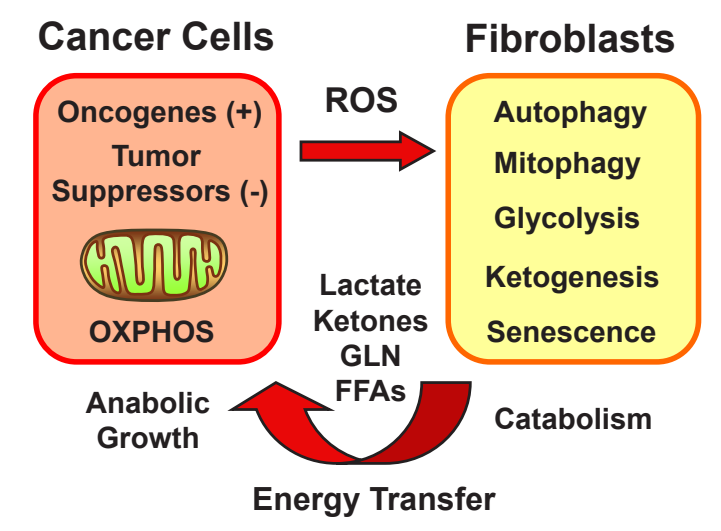

Figure 1: Metabolic-Symbiosis in Human Cancer(s): New Therapeutic Targets. Two-Compartment Tumor Metabolism: Schematic Diagram. Catabolic stromal fibroblasts produce high-energy mitochondrial fuels, for cancer cells to use as substrates for OXPHOS and as biomass. Oncogenes (gainof-function) and tumor suppressors (loss-of-function) both induce catabolism in adjacent fibroblasts, via ROS production (hydrogen peroxide) and the onset of oxidative stress. glycolytic, mitochondrial-poor stromal fibroblasts (Figure 2) [58]. Virtually identical results were also obtained with metabolic protein biomarkers in primary breast tumors and secondary lymph-node metastases (Figure 3), reflecting a common organizing principle, with the juxtaposition of oxidative and glycolytic energetic compartments [52, 59]. As such, tumor architecture also "mirrors" these energybased tumor-stromal interactions.

Remarkably, new studies suggest that normal adjacent epithelial cells, and stromal adipocytes, can also serve as functional metabolic partners for anabolic cancer cells $[22,40,56,60]$. Therefore, cancer cells may be able to use many different cell types, in addition to fibroblasts, as partners to engage in metabolic-symbiosis [52, 61].

Finally, oncogene-transformed epithelial cancer cells also show significant increases in mitochondrial mass, which is strictly dependent on oxidative stress [56]. Figure 4 shows that NAC treatment (N-acetyl-cysteine; an anti-oxidant) dramatically reduced mitochondrial staining, selectively in Ras-transformed cancer cells, but not in matched normal control epithelial cells. Thus, NAC selectively blocks mitochondrial biogenesis in Ras-

\section{Mitochondrial Activity}
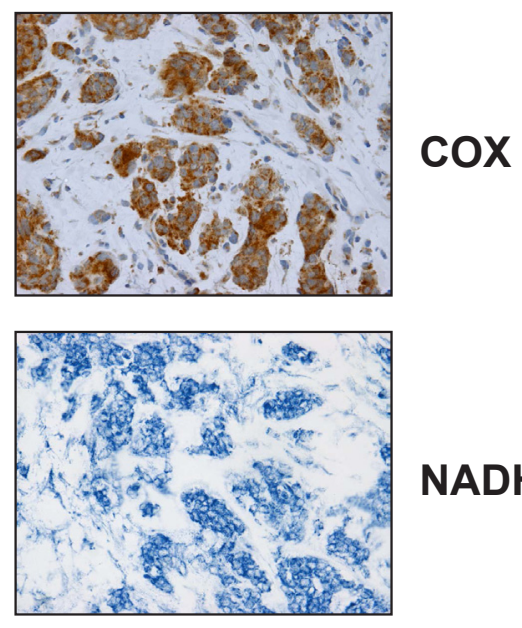

\section{NADH}

$40 \mathrm{X}$

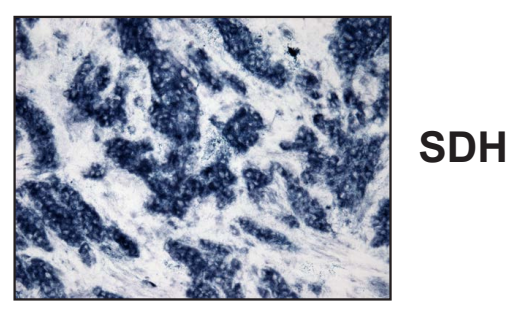

Figure 2: Mitochondrial Activity Staining in Fresh Frozen Human Breast Cancer Tumor Tissue Sections. Note that epithelial cancer cell "nests" amplify their mitochondrial metabolism. In contrast, surrounding stromal fibroblasts show little or no functional mitochondrial staining, indicating that they show a shift towards glycolysis. COX, $\mathrm{NADH}$, and $\mathrm{SBH}$ represent functional activity staining for mitochondrial complex IV, I, and III, respectively. Reproduced, with permission, from [58]. 


\section{MCT4 \& TOMM20}

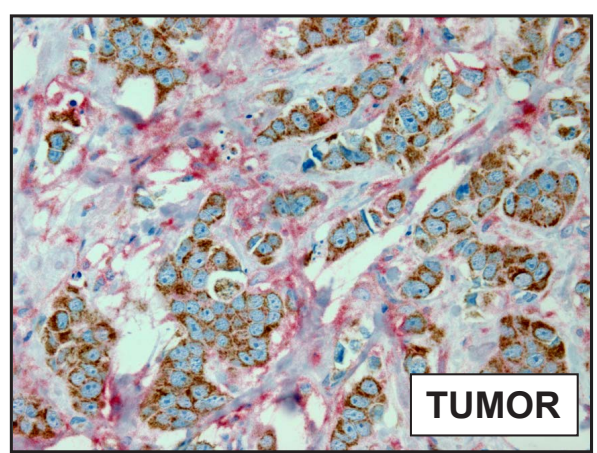

$40 X$
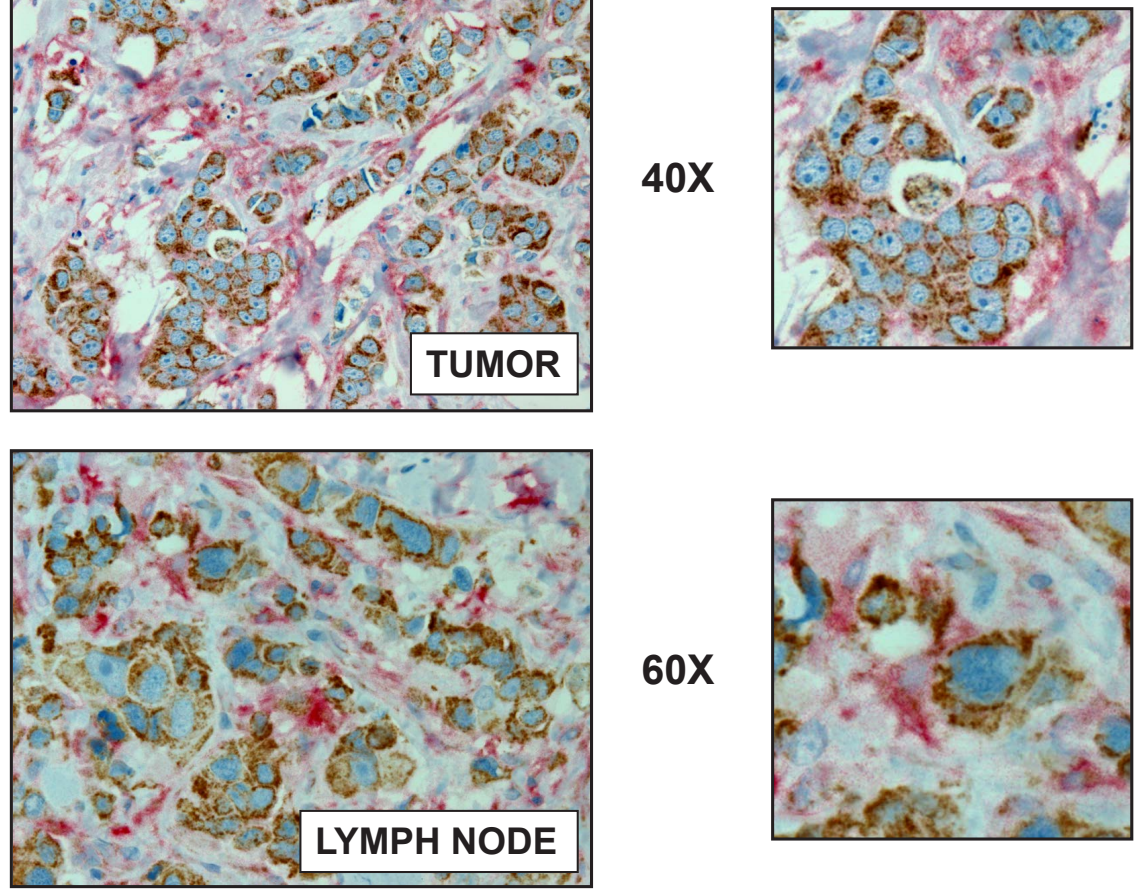

Figure 3: Visualizing Two-Compartment Tumor Metabolism, with Metabolic Marker Proteins: MCT4 and TOMM20. Human breast cancer samples (from primary tumors or lymph node metastases) were immuno-stained with antibodies directed against MCT4 (shown in RED) and TOMM20 (shown in BROWN). MCT4 is a marker of cellular stress, such as ROS production, glycolysis, and mitochondrial dysfunction, which reflects catabolic metabolism in cancer-associated fibroblasts. Conversely, TOMM20 is a marker of mitochondrial mass that has been shown to correlate with oxidative mitochondrial metabolism. Note that two distinct metabolic compartments (oxidative vs. glycolytic) co-exist, side-by-side, in human primary tumors. Virtually identical results were obtained with metastatic breast cancer lesions, within lymph node tissue. Insets are also shown at higher magnification. Reproduced, with permission, from [52].

TOMM20

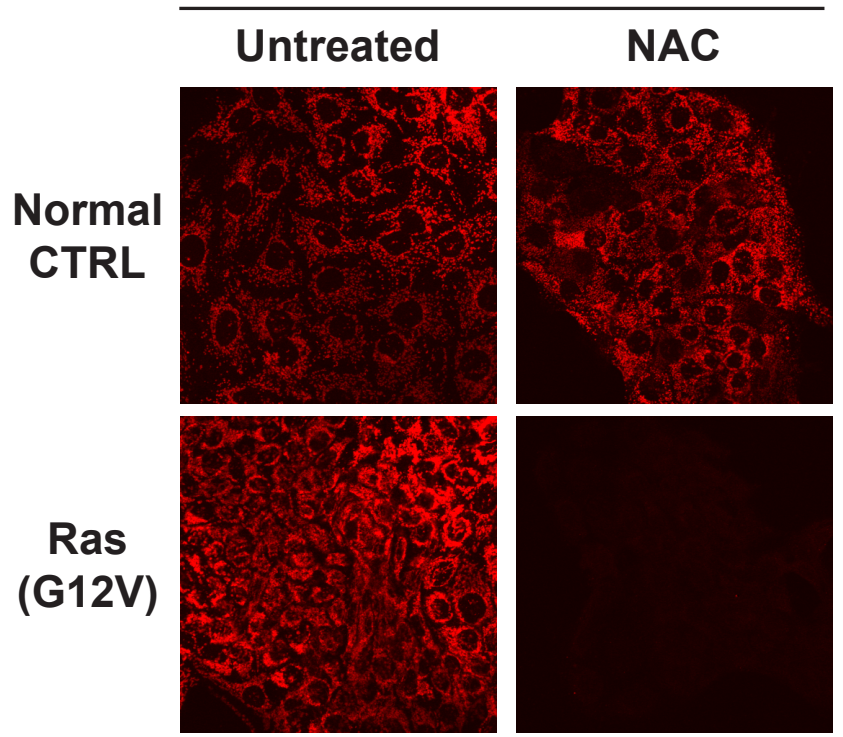

DAPI

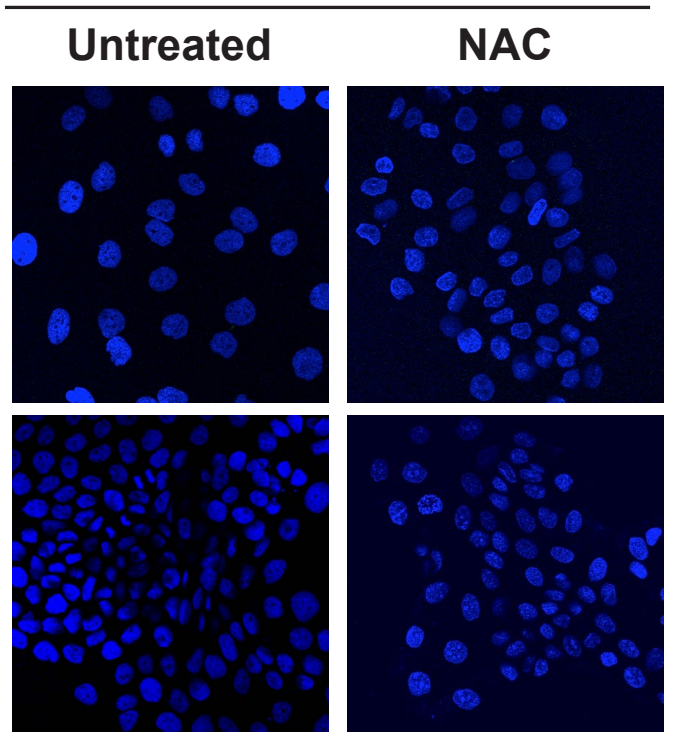

Figure 4: Anti-Oxidants Halt Mitochondrial Biogenesis Selectively in Cancer Cells, But Not in Non-transformed Epithelial Cells. Epithelial cells (control versus H-Ras (G12V) transformed) were maintained (plus or minus NAC (10 mM)) and then subjected to immuno-staining with TOMM20, which is a well-established mitochondrial marker. Note that Ras-transformed cells, treated with NAC, show a significant decrease in mitochondrial mass. DAPI (blue nuclear staining) is also shown. Reproduced, with permission, from $[56]$. 
Table 1: New Validated Targets in Cancer Metabolism

A. Catabolic Cancer-Associated Fibroblasts

Ref.

\begin{tabular}{|l|c|}
\hline Glycolysis/Ketogenesis & {$[1-6]$} \\
\hline CAV1, HIF1A, PKM1, PKM2, CA9, HMGCS2, BDH1, MCT4 & \\
\hline Autophagy/Mitophagy/Inflammation & {$[2,7,8]$} \\
\hline ATG16L1, CTSB, BNIP3, BNIP3L, BECLIN1, NFkB, DRAM, LKB1 & \\
\hline Mitochondrial Dysfunction & {$[9-11]$} \\
\hline TFAM, MFF, UCP1 & \\
\hline Senescence and Cell Cycle Arrest & {$[12]$} \\
\hline p16-INK4A, p19-ARF, p21-CIP1/WAF1 & \\
\hline Growth Factors/Extracelluar Matrix Proteins & {$[13-17]$} \\
\hline $\begin{array}{l}\text { CTGF, TGF-beta1/2/3, TGF-beta type I receptor kinase, Migration Stimulating Factor (MSF), PAI1, PAI2, } \\
\text { PPAR-gamma receptor }\end{array}$ & \\
\hline
\end{tabular}

B. Anabolic Epithelial Cancer Cells

\begin{tabular}{|l|c|}
\hline Mitochondrial Hyper-function & \\
\hline PGC1A, PGC1B, MitoNEET, POLRMT, GOLPH3, HIF2A & {$[8,18,19]$} \\
\hline Lactate and Ketone Metabolism & \\
\hline ACAT1, ACAT2, OXCT1, OXCT2, MCT1 & {$[4,20]$} \\
\hline
\end{tabular}

A. Catabolic fibroblasts were generated by recombinant over-expression or knock-down of key metabolic target genes in hTERT-immortalized fibroblasts. Similarly, catabolic fibroblasts were also obtained by the genetic manipulation of either growth factors or extracellular matrix proteins. Nearly 30 catabolic fibroblast cell lines that have been generated, are listed. Remarkably, these catabolic fibroblasts promoted tumor growth and/or metastasis, in pre-clinical animal models (xenografts in nude mice).

B. Over-expression of genes that drive mitochondrial biogenesis or augment ketone metabolism in MDA-MB-231 epithelial cancer cells, also effectively promoted tumor growth, and induced autophagy-resistance.

transformed cells, illustrating how new drug discovery of more powerful anti-oxidants could be used therapeutically to "starve" cancer cells. Quantitation indicated that the mitochondrial marker TOMM20 was decreased by $>5$-fold during NAC-treatment [56]. As such, oxidative stress and ROS production may drive mitochondrial biogenesis in certain aggressive epithelial cancer cells.

In summary, Table 1 lists $>40$ validated therapeutic target(s), related to metabolic-symbiosis, that could be exploited for new drug discovery. Thus, we should consider metabolic-symbiosis as a novel conceptual framework or platform to design more effective anticancer therapies.

\section{ACKNOWLEDGEMENTS:}

Dr. Ubaldo E. Martinez-Outschoorn was supported by a Young Investigator Award from the Margaret Q. Landenberger Research Foundation.

Dr. Lisanti's and Dr. Sotgia's current affiliation is the University of Manchester (United Kingdom), where they receive funding from the Manchester Cancer Research Centre (MCRC), Breakthrough Breast Cancer (BBC) and
The European Research Council (ERC).

Drs. Michael P. Lisanti and Federica Sotgia were also previously supported by the resources of Thomas Jefferson University in Philadelphia, USA.

\section{REFERENCES}

1. Trimmer C, Sotgia F, Whitaker-Menezes D, Balliet RM, Eaton G, Martinez-Outschoorn UE, Pavlides S, Howell A, Iozzo RV, Pestell RG, Scherer PE, Capozza F and Lisanti MP. Caveolin-1 and mitochondrial SOD2 (MnSOD) function as tumor suppressors in the stromal microenvironment: a new genetically tractable model for human cancer associated fibroblasts. Cancer Biol Ther. 2011; 11(4):383-394.

2. Chiavarina B, Whitaker-Menezes D, Migneco G, MartinezOutschoorn UE, Pavlides S, Howell A, Tanowitz HB, Casimiro MC, Wang C, Pestell RG, Grieshaber P, Caro J, Sotgia F and Lisanti MP. HIF1-alpha functions as a tumor promoter in cancer associated fibroblasts, and as a tumor suppressor in breast cancer cells: Autophagy drives compartment-specific oncogenesis. Cell Cycle. 2010; 9(17):3534-3551. 
3. Chiavarina B, Whitaker-Menezes D, Martinez-Outschoorn UE, Witkiewicz AK, Birbe RC, Howell A, Pestell RG, Smith J, Daniel R, Sotgia F and Lisanti MP. Pyruvate kinase expression (PKM1 and PKM2) in cancer-associated fibroblasts drives stromal nutrient production and tumor growth. Cancer Biol Ther. 2011; 12(12).

4. Martinez-Outschoorn UE, Lin Z, Whitaker-Menezes D, Howell A, Sotgia F and Lisanti MP. Ketone body utilization drives tumor growth and metastasis. Cell Cycle. 2012; 11(21):3964-3971.

5. Whitaker-Menezes D, Martinez-Outschoorn UE, Lin Z, Ertel A, Flomenberg N, Witkiewicz AK, Birbe RC, Howell A, Pavlides S, Gandara R, Pestell RG, Sotgia F, Philp NJ and Lisanti MP. Evidence for a stromal-epithelial "lactate shuttle" in human tumors: MCT4 is a marker of oxidative stress in cancer-associated fibroblasts. Cell Cycle. 2011; 10(11):1772-1783.

6. Fiaschi T, Giannoni E, Taddei ML, Cirri P, Marini A, Pintus G, Nativi C, Richichi B, Scozzafava A, Carta F, Torre E, Supuran CT and Chiarugi P. Carbonic anhydrase IX from cancer-associated fibroblasts drives epithelial-mesenchymal transition in prostate carcinoma cells. Cell Cycle. 2013; 12(11):1791-1801.

7. Capparelli C, Guido C, Whitaker-Menezes D, Bonuccelli G, Balliet R, Pestell TG, Goldberg AF, Pestell RG, Howell A, Sneddon S, Birbe R, Tsirigos A, Martinez-Outschoorn U, Sotgia F and Lisanti MP. Autophagy and senescence in cancer-associated fibroblasts metabolically supports tumor growth and metastasis via glycolysis and ketone production. Cell Cycle. 2012; 11(12):2285-2302.

8. Salem AF, Whitaker-Menezes D, Lin Z, MartinezOutschoorn UE, Tanowitz HB, Al-Zoubi MS, Howell A, Pestell RG, Sotgia F and Lisanti MP. Twocompartment tumor metabolism: autophagy in the tumor microenvironment and oxidative mitochondrial metabolism (OXPHOS) in cancer cells. Cell Cycle. 2012; 11(13):25452556.

9. Balliet RM, Capparelli C, Guido C, Pestell TG, MartinezOutschoorn UE, Lin Z, Whitaker-Menezes D, Chiavarina B, Pestell RG, Howell A, Sotgia F and Lisanti MP. Mitochondrial oxidative stress in cancer-associated fibroblasts drives lactate production, promoting breast cancer tumor growth: understanding the aging and cancer connection. Cell Cycle. 2011; 10(23):4065-4073.

10. Guido C, Whitaker-Menezes D, Lin Z, Pestell RG, Howell A, Zimmers TA, Casimiro MC, Aquila S, Ando S, Martinez-Outschoorn UE, Sotgia F and Lisanti MP. Mitochondrial fission induces glycolytic reprogramming in cancer-associated myofibroblasts, driving stromal lactate production, and early tumor growth. Oncotarget. 2012; 3(8):798-810.

11. Sanchez-Alvarez R, Martinez-Outschoorn UE, Lamb R, Hulit J, Howell A, Gandara R, Sartini M, Rubin E, Lisanti MP and Sotgia F. Mitochondrial dysfunction in breast cancer cells prevents tumor growth: understanding chemoprevention with metformin. Cell Cycle. 2013; 12(1):172-182.

12. Capparelli C, Chiavarina B, Whitaker-Menezes D, Pestell TG, Pestell RG, Hulit J, Ando S, Howell A, MartinezOutschoorn UE, Sotgia F and Lisanti MP. CDK inhibitors (p16/p19/p21) induce senescence and autophagy in cancerassociated fibroblasts, "fueling" tumor growth via paracrine interactions, without an increase in neo-angiogenesis. Cell Cycle. 2012; 11(19):3599-3610.

13. Capparelli C, Whitaker-Menezes D, Guido C, Balliet R, Pestell TG, Howell A, Sneddon S, Pestell RG, MartinezOutschoorn U, Lisanti MP and Sotgia F. CTGF drives autophagy, glycolysis and senescence in cancer-associated fibroblasts via HIF1 activation, metabolically promoting tumor growth. Cell Cycle. 2012; 11(12):2272-2284.

14. Guido C, Whitaker-Menezes D, Capparelli C, Balliet R, Lin Z, Pestell RG, Howell A, Aquila S, Ando S, MartinezOutschoorn U, Sotgia F and Lisanti MP. Metabolic reprogramming of cancer-associated fibroblasts by TGFbeta drives tumor growth: Connecting TGF-beta signaling with "Warburg-like" cancer metabolism and L-lactate production. Cell Cycle. 2012; 11(16):3019-3035.

15. Carito V, Bonuccelli G, Martinez-Outschoorn UE, Whitaker-Menezes D, Caroleo MC, Cione E, Howell A, Pestell RG, Lisanti MP and Sotgia F. Metabolic remodeling of the tumor microenvironment: Migration stimulating factor (MSF) reprograms myofibroblasts toward lactate production, fueling anabolic tumor growth. Cell Cycle. 2012; 11(18):3403-3414.

16. Castello-Cros R, Bonuccelli G, Molchansky A, Capozza F, Witkiewicz AK, Birbe RC, Howell A, Pestell RG, Whitaker-Menezes D, Sotgia F and Lisanti MP. Matrix remodeling stimulates stromal autophagy, "fueling" cancer cell mitochondrial metabolism and metastasis. Cell Cycle. 2011; 10(12):2021-2034.

17. Avena $\mathrm{P}$, Anselmo W, Whitaker-Menezes D, Wang C, Pestell RG, Lamb RS, Hulit J, Casaburi I, Ando S, Martinez-Outschoorn UE, Lisanti MP and Sotgia F. Compartment-specific activation of PPARgamma governs breast cancer tumor growth, via metabolic reprogramming and symbiosis. Cell Cycle. 2013; 12(9):1360-1370.

18. Salem AF, Whitaker-Menezes D, Howell A, Sotgia F and Lisanti MP. Mitochondrial biogenesis in epithelial cancer cells promotes breast cancer tumor growth and confers autophagy resistance. Cell Cycle. 2012; 11(23):4174-4180.

19. Chiavarina B, Martinez-Outschoorn UE, Whitaker-Menezes D, Howell A, Tanowitz HB, Pestell RG, Sotgia F and Lisanti MP. Metabolic reprogramming and two-compartment tumor metabolism: Opposing role(s) of HIF1alpha and HIF2alpha in tumor-associated fibroblasts and human breast cancer cells. Cell Cycle. 2012; 11(17):3280-3289.

20. Fiaschi T, Marini A, Giannoni E, Taddei ML, Gandellini P, De Donatis A, Lanciotti M, Serni S, Cirri P and Chiarugi P. Reciprocal Metabolic Reprogramming through Lactate Shuttle Coordinately Influences Tumor-Stroma Interplay. 
Cancer Res. 2012.

21. Brauer HA, Makowski L, Hoadley KA, Casbas-Hernandez P, Lang LJ, Roman-Perez E, D'Arcy M, Freemerman $\mathrm{AJ}$, Perou $\mathrm{CM}$ and Troester MA. Impact of tumor microenvironment and epithelial phenotypes on metabolism in breast cancer. Clin Cancer Res. 2013; 19(3):571-585.

22. Nieman KM, Kenny HA, Penicka CV, Ladanyi A, BuellGutbrod R, Zillhardt MR, Romero IL, Carey MS, Mills GB, Hotamisligil GS, Yamada SD, Peter ME, Gwin K and Lengyel E. Adipocytes promote ovarian cancer metastasis and provide energy for rapid tumor growth. Nat Med. 2011; 17(11):1498-1503.

23. Fordyce CA, Patten KT, Fessenden TB, Defilippis R, Hwang ES, Zhao J and Tlsty TD. Cell-extrinsic consequences of epithelial stress: activation of protumorigenic tissue phenotypes. Breast Cancer Res. 2012; 14(6):R155.

24. Chaudhri VK, Salzler GG, Dick SA, Buckman MS, Sordella R, Karoly ED, Mohney R, Stiles BM, Elemento O, Altorki NK and McGraw TE. Metabolic Alterations in Lung Cancer-Associated Fibroblasts Correlated with Increased Glycolytic Metabolism of the Tumor. Mol Cancer Res. 2013.

25. Lisanti MP, Martinez-Outschoorn UE, Chiavarina B, Pavlides S, Whitaker-Menezes D, Tsirigos A, Witkiewicz A, Lin Z, Balliet R, Howell A and Sotgia F. Understanding the "lethal" drivers of tumor-stroma co-evolution: emerging role(s) for hypoxia, oxidative stress and autophagy/ mitophagy in the tumor micro-environment. Cancer Biol Ther. 2010; 10(6):537-542.

26. Lisanti MP, Martinez-Outschoorn UE, Lin Z, Pavlides S, Whitaker-Menezes D, Pestell RG, Howell A and Sotgia F. Hydrogen peroxide fuels aging, inflammation, cancer metabolism and metastasis: the seed and soil also needs “fertilizer". Cell Cycle. 2011; 10(15):2440-2449.

27. Lisanti MP, Martinez-Outschoorn UE, Pavlides S, Whitaker-Menezes D, Pestell RG, Howell A and Sotgia F. Accelerated aging in the tumor microenvironment: connecting aging, inflammation and cancer metabolism with personalized medicine. Cell Cycle. 2011; 10(13):20592063.

28. Martinez-Outschoorn UE, Balliet R, Lin Z, WhitakerMenezes D, Birbe RC, Bombonati A, Pavlides S, Lamb R, Sneddon S, Howell A, Sotgia F and Lisanti MP. BRCA1 mutations drive oxidative stress and glycolysis in the tumor microenvironment: implications for breast cancer prevention with antioxidant therapies. Cell Cycle. 2012; 11(23):4402-4413.

29. Martinez-Outschoorn UE, Balliet RM, Lin Z, WhitakerMenezes D, Howell A, Sotgia F and Lisanti MP. Hereditary ovarian cancer and two-compartment tumor metabolism: epithelial loss of BRCA1 induces hydrogen peroxide production, driving oxidative stress and NFkappaB activation in the tumor stroma. Cell Cycle. 2012; 11(22):4152-4166.

30. Martinez-Outschoorn UE, Balliet RM, Rivadeneira DB,
Chiavarina B, Pavlides S, Wang C, Whitaker-Menezes D, Daumer KM, Lin Z, Witkiewicz AK, Flomenberg N, Howell A, Pestell RG, Knudsen ES, Sotgia F and Lisanti MP. Oxidative stress in cancer associated fibroblasts drives tumor-stroma co-evolution: A new paradigm for understanding tumor metabolism, the field effect and genomic instability in cancer cells. Cell Cycle. 2010; 9(16):3256-3276.

31. Martinez-Outschoorn UE, Goldberg A, Lin Z, Ko YH, Flomenberg N, Wang C, Pavlides S, Pestell RG, Howell A, Sotgia $\mathrm{F}$ and Lisanti MP. Anti-estrogen resistance in breast cancer is induced by the tumor microenvironment and can be overcome by inhibiting mitochondrial function in epithelial cancer cells. Cancer Biol Ther. 2011; 12(10):924938.

32. Martinez-Outschoorn UE, Lin Z, Ko YH, Goldberg AF, Flomenberg N, Wang C, Pavlides S, Pestell RG, Howell A, Sotgia $\mathrm{F}$ and Lisanti MP. Understanding the metabolic basis of drug resistance: therapeutic induction of the Warburg effect kills cancer cells. Cell Cycle. 2011; 10(15):25212528.

33. Martinez-Outschoorn UE, Lin Z, Trimmer C, Flomenberg N, Wang C, Pavlides S, Pestell RG, Howell A, Sotgia F and Lisanti MP. Cancer cells metabolically "fertilize" the tumor microenvironment with hydrogen peroxide, driving the Warburg effect: implications for PET imaging of human tumors. Cell Cycle. 2010; 10(15):2504-2520.

34. Martinez-Outschoorn UE, Lin Z, Whitaker-Menezes D, Howell A, Lisanti MP and Sotgia F. Ketone bodies and twocompartment tumor metabolism: Stromal ketone production fuels mitochondrial biogenesis in epithelial cancer cells. Cell Cycle. 2012; 11(21).

35. Martinez-Outschoorn UE, Pavlides S, Howell A, Pestell RG, Tanowitz HB, Sotgia F and Lisanti MP. Stromalepithelial metabolic coupling in cancer: integrating autophagy and metabolism in the tumor microenvironment. Int J Biochem Cell Biol. 2011; 43(7):1045-1051.

36. Martinez-Outschoorn UE, Pavlides S, Sotgia F and Lisanti MP. Mitochondrial biogenesis drives tumor cell proliferation. Am J Pathol. 2011; 178(5):1949-1952.

37. Martinez-Outschoorn UE, Pavlides S, Whitaker-Menezes D, Daumer KM, Milliman JN, Chiavarina B, Migneco G, Witkiewicz AK, Martinez-Cantarin MP, Flomenberg N, Howell A, Pestell RG, Lisanti MP and Sotgia F. Tumor cells induce the cancer associated fibroblast phenotype via caveolin-1 degradation: implications for breast cancer and DCIS therapy with autophagy inhibitors. Cell Cycle. 2010; 9(12):2423-2433.

38. Martinez-Outschoorn UE, Pestell RG, Howell A, Tykocinski ML, Nagajyothi F, Machado FS, Tanowitz HB, Sotgia F and Lisanti MP. Energy transfer in "parasitic" cancer metabolism: mitochondria are the powerhouse and Achilles' heel of tumor cells. Cell Cycle. 2011; 10(24):4208-4216.

39. Martinez-Outschoorn UE, Prisco M, Ertel A, Tsirigos A, 
Lin Z, Pavlides S, Wang C, Flomenberg N, Knudsen ES, Howell A, Pestell RG, Sotgia F and Lisanti MP. Ketones and lactate increase cancer cell "stemness," driving recurrence, metastasis and poor clinical outcome in breast cancer: achieving personalized medicine via MetaboloGenomics. Cell Cycle. 2011; 10(8):1271-1286.

40. Martinez-Outschoorn UE, Sotgia F and Lisanti MP. Power surge: supporting cells "fuel" cancer cell mitochondria. Cell Metab. 2012; 15(1):4-5.

41. Martinez-Outschoorn UE, Trimmer C, Lin Z, WhitakerMenezes D, Chiavarina B, Zhou J, Wang C, Pavlides S, Martinez-Cantarin MP, Capozza F, Witkiewicz AK, Flomenberg N, Howell A, Pestell RG, Caro J, Lisanti MP, et al. Autophagy in cancer associated fibroblasts promotes tumor cell survival: Role of hypoxia, HIF1 induction and NFkappaB activation in the tumor stromal microenvironment. Cell Cycle. 2010; 9(17):3515-3533.

42. Martinez-Outschoorn UE, Whitaker-Menezes D, Lin Z, Flomenberg N, Howell A, Pestell RG, Lisanti MP and Sotgia F. Cytokine production and inflammation drive autophagy in the tumor microenvironment: role of stromal caveolin-1 as a key regulator. Cell Cycle. 2011; 10(11):1784-1793.

43. Martinez-Outschoorn UE, Whitaker-Menezes D, Pavlides S, Chiavarina B, Bonuccelli G, Casey T, Tsirigos A, Migneco G, Witkiewicz A, Balliet R, Mercier I, Wang C, Flomenberg N, Howell A, Lin Z, Caro J, et al. The autophagic tumor stroma model of cancer or "batteryoperated tumor growth": A simple solution to the autophagy paradox. Cell Cycle. 2010; 9(21):4297-4306.

44. Pavlides S, Tsirigos A, Migneco G, Whitaker-Menezes D, Chiavarina B, Flomenberg N, Frank PG, Casimiro MC, Wang C, Pestell RG, Martinez-Outschoorn UE, Howell A, Sotgia F and Lisanti MP. The autophagic tumor stroma model of cancer: Role of oxidative stress and ketone production in fueling tumor cell metabolism. Cell Cycle. 2010; 9(17):3485-3505.

45. Pavlides S, Tsirigos A, Vera I, Flomenberg N, Frank PG, Casimiro MC, Wang C, Fortina P, Addya S, Pestell RG, Martinez-Outschoorn UE, Sotgia F and Lisanti MP. Loss of stromal caveolin-1 leads to oxidative stress, mimics hypoxia and drives inflammation in the tumor microenvironment, conferring the "reverse Warburg effect": a transcriptional informatics analysis with validation. Cell Cycle. 2010; 9(11):2201-2219.

46. Pavlides S, Tsirigos A, Vera I, Flomenberg N, Frank PG, Casimiro MC, Wang C, Pestell RG, Martinez-Outschoorn UE, Howell A, Sotgia F and Lisanti MP. Transcriptional evidence for the "Reverse Warburg Effect" in human breast cancer tumor stroma and metastasis: similarities with oxidative stress, inflammation, Alzheimer's disease, and "Neuron-Glia Metabolic Coupling". Aging (Albany NY). 2010; 2(4):185-199.

47. Pavlides S, Vera I, Gandara R, Sneddon S, Pestell RG, Mercier I, Martinez-Outschoorn UE, Whitaker-Menezes
D, Howell A, Sotgia F and Lisanti MP. Warburg meets autophagy: cancer-associated fibroblasts accelerate tumor growth and metastasis via oxidative stress, mitophagy, and aerobic glycolysis. Antioxid Redox Signal. 2012; 16(11):1264-1284.

48. Pavlides S, Whitaker-Menezes D, Castello-Cros R, Flomenberg N, Witkiewicz AK, Frank PG, Casimiro MC, Wang C, Fortina P, Addya S, Pestell RG, MartinezOutschoorn UE, Sotgia F and Lisanti MP. The reverse Warburg effect: aerobic glycolysis in cancer associated fibroblasts and the tumor stroma. Cell Cycle. 2009; 8(23):3984-4001.

49. Sotgia F, Martinez-Outschoorn UE, Howell A, Pestell RG, Pavlides S and Lisanti MP. Caveolin-1 and cancer metabolism in the tumor microenvironment: markers, models, and mechanisms. Annu Rev Pathol. 2012; 7:423467.

50. Sotgia F, Martinez-Outschoorn UE and Lisanti MP. Mitochondrial oxidative stress drives tumor progression and metastasis: should we use antioxidants as a key component of cancer treatment and prevention? BMC Med. 2011; 9:62.

51. Sotgia F, Martinez-Outschoorn UE, Pavlides S, Howell A, Pestell RG and Lisanti MP. Understanding the Warburg effect and the prognostic value of stromal caveolin-1 as a marker of a lethal tumor microenvironment. Breast Cancer Res. 2011; 13(4):213.

52. Sotgia F, Whitaker-Menezes D, Martinez-Outschoorn UE, Flomenberg N, Birbe RC, Witkiewicz AK, Howell A, Philp NJ, Pestell RG and Lisanti MP. Mitochondrial metabolism in cancer metastasis: visualizing tumor cell mitochondria and the "reverse Warburg effect" in positive lymph node tissue. Cell Cycle. 2012; 11(7):1445-1454.

53. Migneco G, Whitaker-Menezes D, Chiavarina B, CastelloCros R, Pavlides S, Pestell RG, Fatatis A, Flomenberg N, Tsirigos A, Howell A, Martinez-Outschoorn UE, Sotgia F and Lisanti MP. Glycolytic cancer associated fibroblasts promote breast cancer tumor growth, without a measurable increase in angiogenesis: evidence for stromal-epithelial metabolic coupling. Cell Cycle. 2010; 9(12):2412-2422.

54. Witkiewicz AK, Dasgupta A, Nguyen KH, Liu C, Kovatich AJ, Schwartz GF, Pestell RG, Sotgia F, Rui H and Lisanti MP. Stromal caveolin-1 levels predict early DCIS progression to invasive breast cancer. Cancer Biol Ther. 2009; 8(11):1071-1079.

55. Hanahan D and Coussens LM. Accessories to the crime: functions of cells recruited to the tumor microenvironment. Cancer Cell. 2012; 21(3):309-322.

56. Martinez-Outschoorn UE, Curry JM, Ko Y-H, Lin Z, Tuluc M, Cognetti D, Birbe R, Pribitkin E, Bombonati A, Pestell RG, Howell A, Sotgia F and Lisanti MP. Oncogenes and inflammation rewire host energy metabolism in the tumor microenvironment: RAS and NFkB target stromal MCT4. Cell Cycle. 2013; 12(16):In Press.

57. Mercier I, Camacho J, Titchen K, Gonzales DM, Quann K, Bryant KG, Molchansky A, Milliman JN, 
Whitaker-Menezes D, Sotgia F, Jasmin JF, Schwarting R, Pestell RG, Blagosklonny MV and Lisanti MP. Caveolin-1 and accelerated host aging in the breast tumor microenvironment: chemoprevention with rapamycin, an mTOR inhibitor and anti-aging drug. Am J Pathol. 2012; 181(1):278-293.

58. Whitaker-Menezes D, Martinez-Outschoorn UE, Flomenberg N, Birbe RC, Witkiewicz AK, Howell A, Pavlides S, Tsirigos A, Ertel A, Pestell RG, Broda P, Minetti C, Lisanti MP and Sotgia F. Hyperactivation of oxidative mitochondrial metabolism in epithelial cancer cells in situ: visualizing the therapeutic effects of metformin in tumor tissue. Cell Cycle. 2011; 10(23):4047-4064.

59. Sotgia F, Whitaker-Menezes D, Martinez-Outschoorn UE, Salem AF, Tsirigos A, Lamb R, Sneddon S, Hulit J, Howell A and Lisanti MP. Mitochondria "fuel" breast cancer metabolism: fifteen markers of mitochondrial biogenesis label epithelial cancer cells, but are excluded from adjacent stromal cells. Cell Cycle. 2012; 11(23):4390-4401.

60. Lisanti MP, Martinez-Outschoorn UE and Sotgia F. Oncogenes induce the cancer-associated fibroblast phenotype: Metabolic symbiosis and "fibroblast addiction" are new therapeutic targets for drug discovery. Cell Cycle. 2013; 12(17):in Press.

61. Curry JM, Tuluc M, Whitaker-Menezes D, Ames JA, Anantharaman A, Butera A, Leiby B, Cognetti DM, Sotgia F, Lisanti MP and Martinez-Outschoorn UE. Cancer metabolism, stemness and tumor recurrence: MCT1 and MCT4 are functional biomarkers of metabolic symbiosis in head and neck cancer. Cell Cycle. 2013; 12(9):1371-1384. 